\title{
USE OF FATIGUE ANALYSIS SOFTWARE IN THE OPTIMIZATION OF STRUCTURAL APPROVAL PROCESS FOR VEHICLE COMPONENTS
}

\author{
Scozzafave C.C., Ericsson L. G. S., Santana F.A., Wildmann U., Spinelli D. M. \\ Mercedes-Benz do Brasil \\ E-mails: caio.scozzafave@daimler.com, luis.ericsson@daimler.com \\ flavio_arcanjo.santana@daimler.com, udo.wildmann@daimler.com \\ daniel.spinelli@daimler.com
}

\begin{abstract}
This paper aims to propose a methodology for optimizing the approval process of vehicle components introducing the fatigue analysis via software. The case of study used for methodology application was a commercial vehicle suspension part. The commercial vehicle was modeled in finite element for static stress analysis in several load cases. Durability tests in proving ground with measurements were also done. The component has failed without reaching the track approval criteria. With the measurement signal of force, a fatigue analysis was done correlating the obtained damage with the covered distance in test field until component failure. A new support proposal was modeled and analyzed using finite elements. The optimization of structural approval process comes by eliminating the new version test at proving ground, approving it by force measurement correlation of the first version with the second version, and new fatigue analysis via software. The optimization process reduces the time and cost during product development.
\end{abstract}

\section{INTRODUCTION}

The modern development process for automotive components aims a reduction of time in components tests, due the necessity of acceleration in parts approval process for faster vehicle sign off delivery. The process becomes challenging because despite of the decrease for test time, the product quality and reliability still has to be ensured.

The product development time reduction can be aided by deeper analyses in component homologation methods via simulation, since it can reduce a significant time and cost consuming during the development process. The present study aims to present a methodology to accelerate a component homologation via FEA and fatigue analysis.

Fatigue is the process of damage and failure due to cyclic loading, even when this loading causes stresses well below a given material's ultimate strength, since the microscopic damage can be accumulated until crack nucleation leading to further component failure [1].

The proposed method has its basis on several topics that are covered in this paper, such as: finite element analysis (FEA), fatigue analysis with FEA, signal measurement, signal analysis and accelerated durability components test. Since fatigue analysis is the center of the study, an 
emphasis is given to this topic, and the state of art for some current fatigue hot topics are presented, such as: cumulative damage analysis, local plastification, multiaxial fatigue and also critical planes approach.

Finally, this papers follows the same sequence of product development, which is component design, FEA analysis and further durability test, going through damage simulation and correlation, ending with new component design and approval method description.

\section{FEA ANALYSIS}

To start the component analysis, its design was done and modeled in FEM, as can be seen in figure 1. For this first component version, solid elements were used in the modeling due the necessity of more refined results.

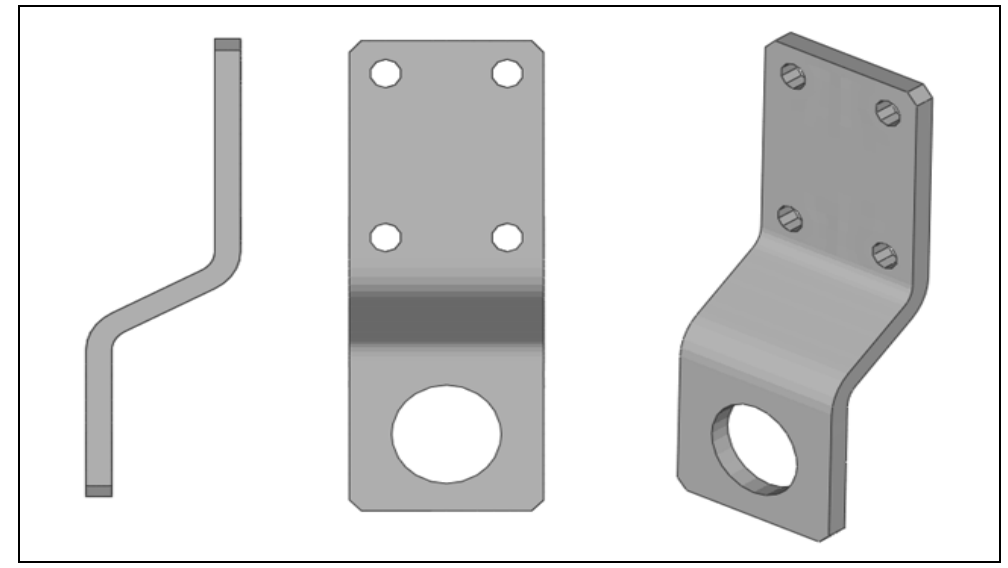

Figure 1. Component FEA model (first version)

A full FEA model of the complete vehicle was also modeled; a picture of the model is presented in figure 2 . The vehicle parts are mainly modeled using shell elements, which gives a good relationship between stress results and simulation time consumption [2]. Only a few parts were modeled in solid elements, such as the component studied.

The full vehicle was simulated in the most severe load case for this component, which is the vehicle torsion. The main stress result that is found in the component is shown in figure 3. At the most critical region it the torsion analysis, the stress reached $100 \%$ of the material limit.

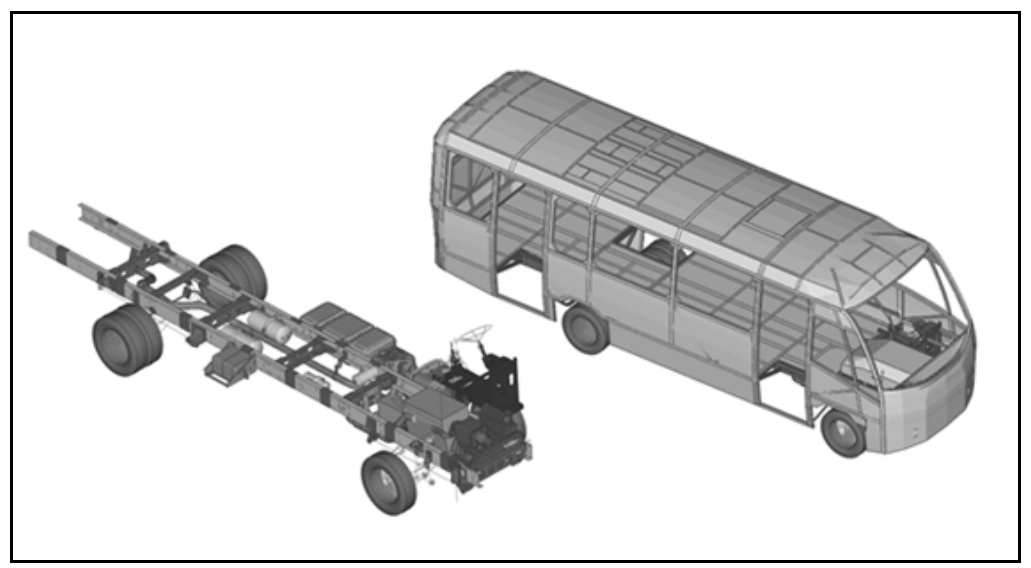

Figure 2. Full FEA model of the complete vehicle 


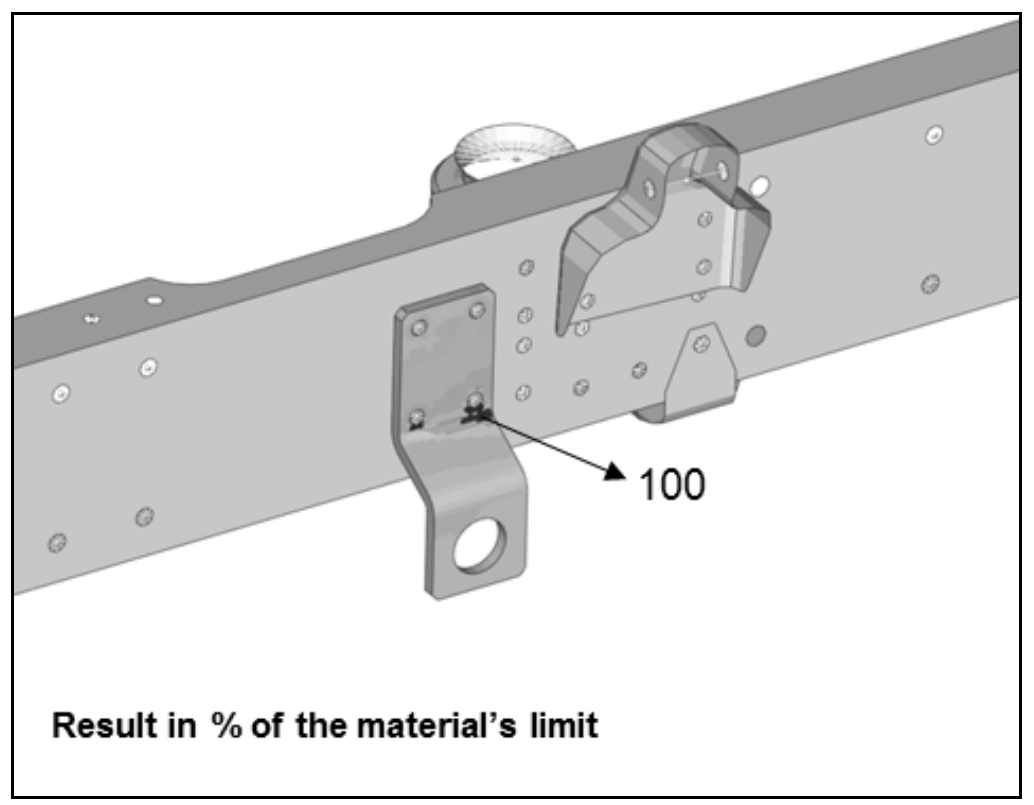

Figure 3. Stress results for torsion load case

\section{COMPONENT DURABILITY TEST}

The next step for product homologation is the durability test. The complete vehicle, that is showed in figure 4 is tested on an accelerated durability proving ground, with pre-established approval criteria for all components of 2940 laps.

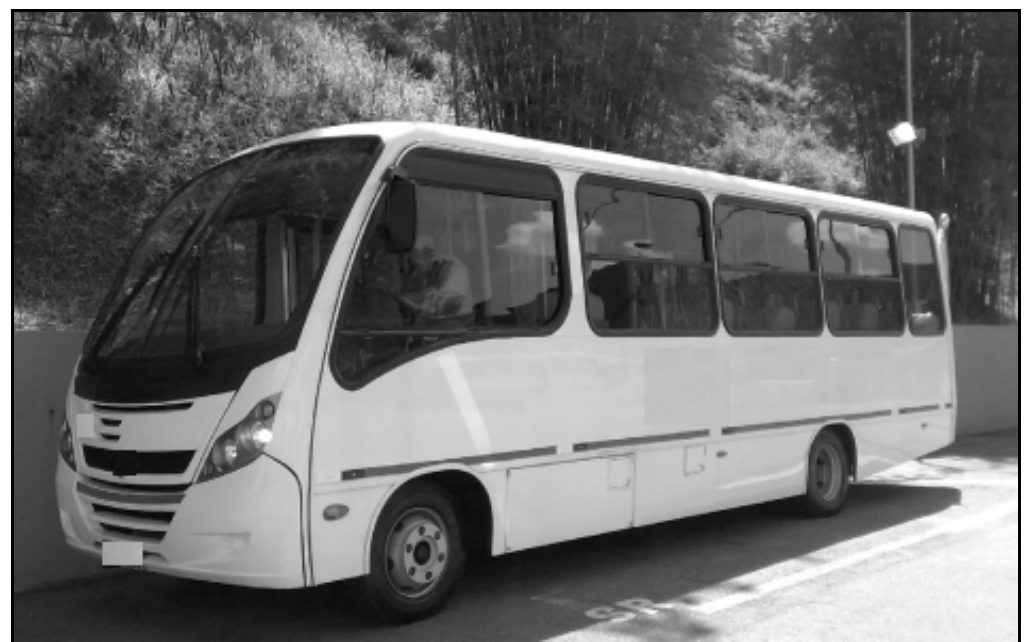

Figure 4. Complete vehicle for durability test

In the beginning of the test, measurement of the load that is applied in the component is done, to make possible the correlation of the load measured in the torture track with the FEA load case criteria, as well as, its stress results and further damage analysis. Figure 5 shows the component to be mounted in the vehicle and the equipment used to measure the force that is applied in the part during the vehicle test at proving ground.

The vehicle is tested part of the time loaded and part of the time unloaded, until it reaches the approval criteria (2940 laps). Both of the situations need to be measured to a better result correlation. In figures 6 and 7 it is presented the force measurement for two laps in both cases. 


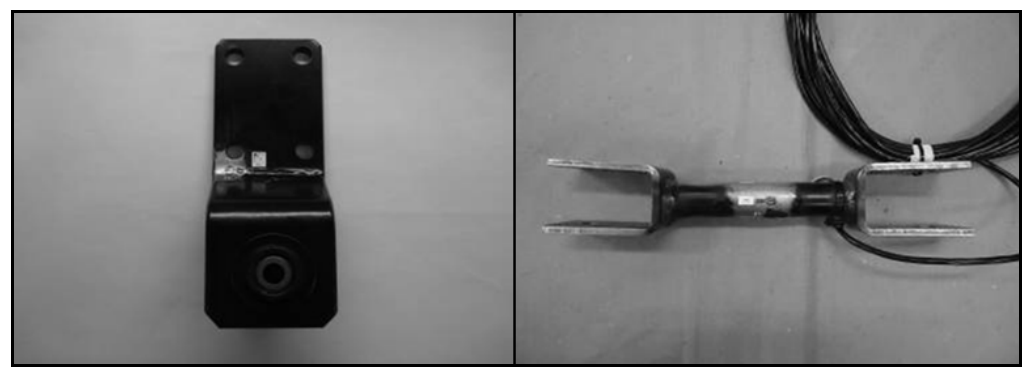

Figure 5. Component first version and equipment used to force measurement

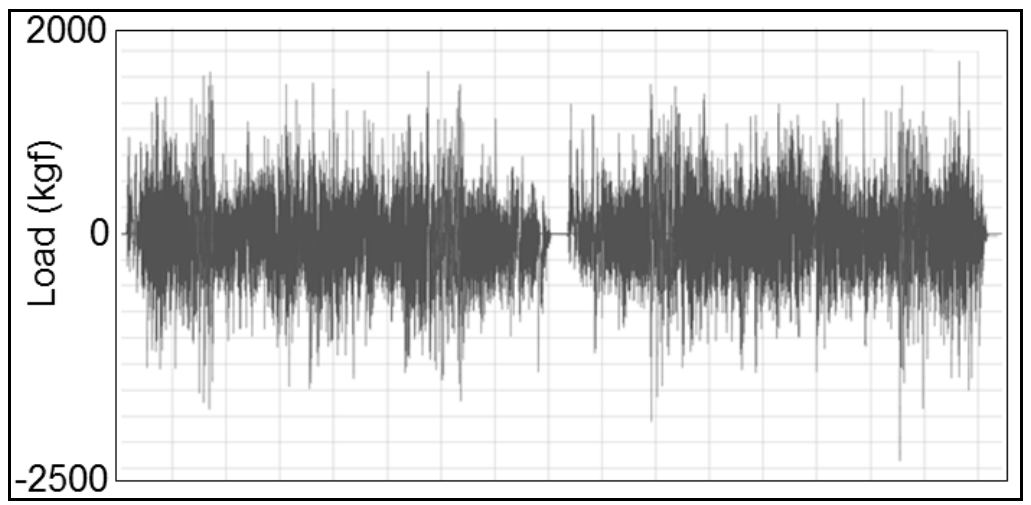

Figure 6. Force measurement at loaded condition

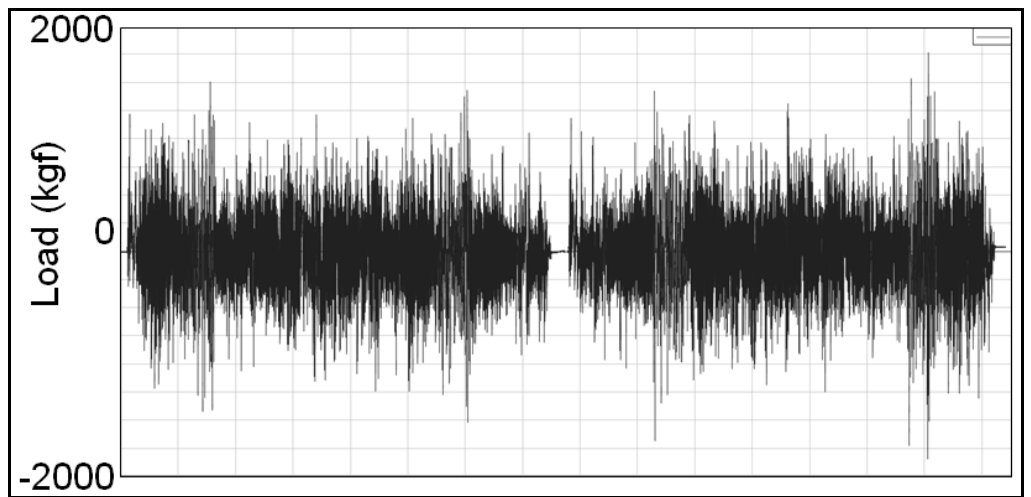

Figure 7. Force measurement at unloaded contidion

\section{FATIGUE ANALYSIS}

\subsection{Cumulative Damage}

In the fatigue analysis field, the Miner linear damage accumulation hypothesis [3] is the most acceptable method for estimating life time by damage accumulation

$$
D=\sum_{i=1} D_{i}=\sum_{i=1} \frac{n_{i}}{N_{i}}
$$

Where:

$D=$ Total damage

$D_{i}=$ Damage for each different stress $\sigma_{i}$ 
$n_{i}=$ Number of cycles that a component is subjected in a given alternating stress $\sigma_{i}$.

$N_{i}=$ Number of cycles to failure at $\sigma_{i}$, according to component material's Wöhler curve.

The individual damage for each different $\sigma_{i}$ are totalized in the course of the fatigue life estimate. When a damage total $\mathrm{D}=1$ is reached, component failure occurs.

\subsection{Plastification in fatigue analysis}

Whenever the stress exceeds the material's yield stress, plastification occurs. The linear stresscurve is adopted for all stress cycles. The displacement of the closed hysteresis loops caused by the plastification is then taken into consideration. Figure 8 shows this procedure. Transformation of the elastic stress to the elastic-plastic stress is given by the Neuber rule [4].

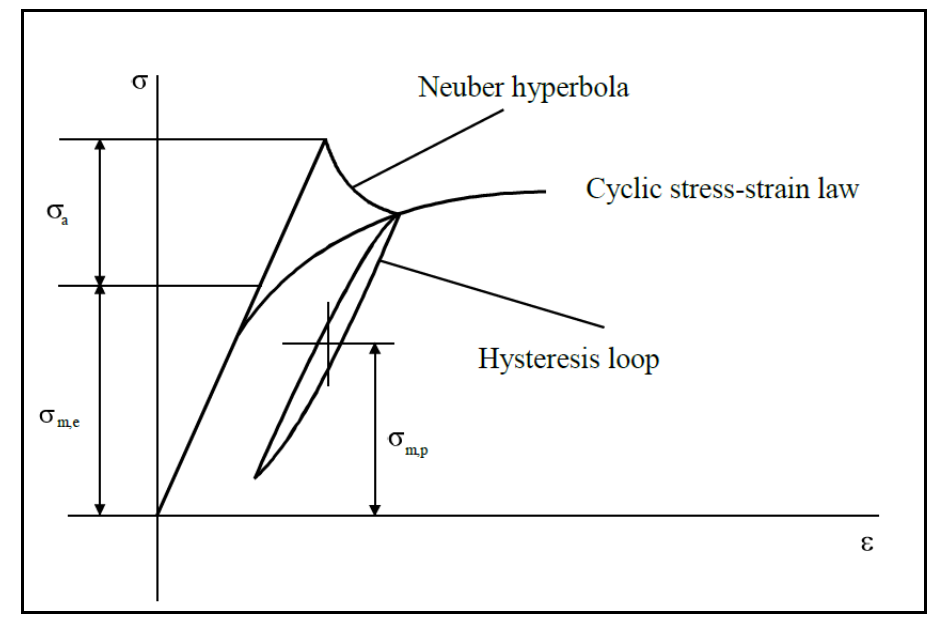

Figure 8. Neuber correction and plastification effect in cyclic stress

\subsection{Multiaxial Fatigue}

Multi-axial loadings are common in various components and structures. By the combination of multi-axial loadings, the fatigue analysis goes from uniaxial approach to more sophisticated multiaxial approach [5].

For components subjected to direct stress and shear, the phase relationship between the stresses cannot be constant. In this case, it is not obvious which plan experiences the most severe combination of deformation and hence greater fatigue damage. The orientation of a plane is defined by the angle that the normal to the plane makes with the xyz coordinate system oriented along the surface. The plan has an angle $\Phi$ of the x-axis towards the $y$ axis [6].

When loading is significantly no proportional, an analysis by critical planes is required, where stresses and strains are determined for the various planes of orientation in the material during cyclic loading, and the stresses and strains acting in the more critically loaded is used to predict the damage [6]. An example is shown in figure 9. 


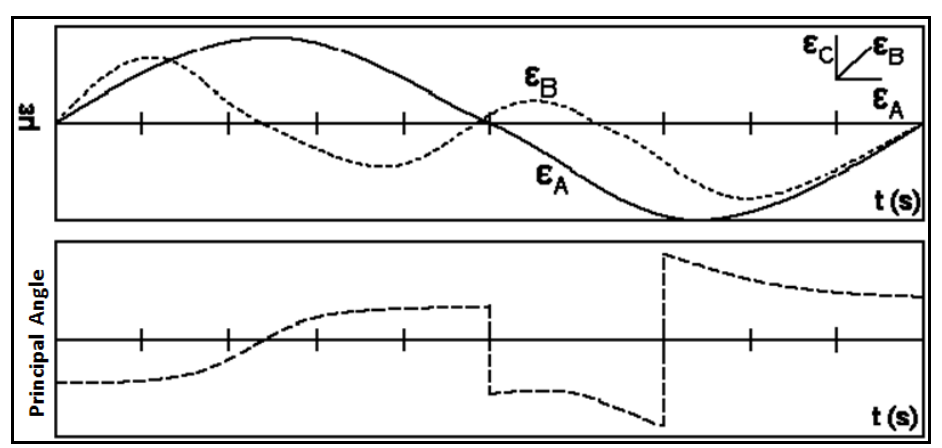

Figure 9. No proportional example [6].

\subsection{Critical plane approach}

Critical plane approaches assumes that fatigue damage is essentially directional and so consider the damage accumulation on several planes. The critical plane is the plane on which fatigue strength assessment is to be performed. A range of methods for calculating damage on a particular plane is described in the literature. The method considered in this paper consists of the normal stress use in each plane for fatigue analysis [7].

The critical plane criterion method consist of: In each plane a rainflow counting of the normal stress history is performed. The resulting stress amplitudes can be used together with a tension/compression S-N curve for the calculation and linear summation of partial damages according to Palmgren/Miner. Before damage analysis the $\mathrm{S}-\mathrm{N}$ curve should be locally modified according to influences like mean stress (which can be quantified by means of an Haigh-diagram), notch support effect (which can be considered by taking into account stress gradient [8]), temperature, surface roughness, surface treatments, etc. The plane with maximum damage is assumed to be critical. The ductility of the material has already been taken into account by scaling of the normal stress or stress tensor [7].

\subsection{Fatigue analysis of the component}

Sub items 3.1, 3.2, 3.3 and 3.4 are taken into account to start the fatigue analysis of the component, because:

1. Variable amplitude of load is encountered, so cumulative damage analysis is required.

2. By FEA analysis, high stresses can be observed, so a local plastification in the critical region is possible.

3. For the uncertainty of the stress/strain proportionality at the component, a multiaxial fatigue approach has to be used, and the chosen one is the critical plane approach.

To do the fatigue analysis of the component, a reduced FEA model is created. Since figure 6 and 7 represents the real load input in the component, the torsion load case is no more necessary. The torsion load case is a static analysis for maximum torsion, but for a fatigue analysis, a simulation with variable load input is required. Now, the load from the measurement is directly inputted in the model, using the FEA model and post-processor software of fatigue analysis. The reduced model, as well as, the location of the load input is showed in figure 10 .. 


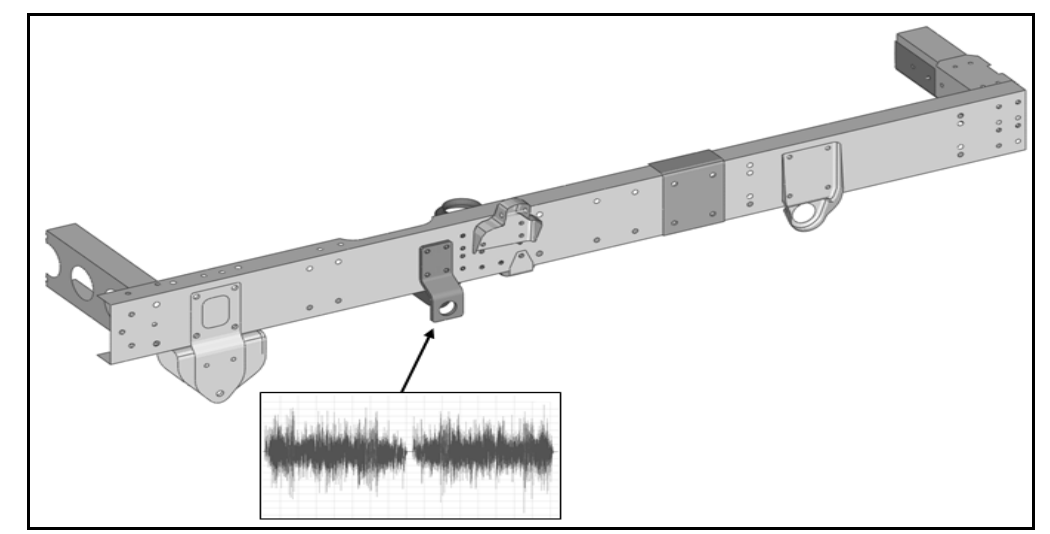

Figure 10. Reduced model and variable load input location

With the reduced model and variable load input showed in figure 6,7, and 10, the fatigue analysis can be done for this part version. The parameters of the analysis, as well as, its results can be seen in figure 11. It is important to notice that in this analysis, the 4 laps force measurement is the load input, and the laps are extrapolated until theoretical component failure. Also, the software takes into account several factors, such as: mean stress, surface roughness, surface treatments, thermal influence, statistical material data, etc.

\begin{tabular}{|l|l|l|}
\hline \multirow{2}{*}{} & \multicolumn{2}{|c|}{ Fatigue analysis } \\
\cline { 2 - 3 } & Load input: & $\begin{array}{l}4 \text { laps extrapolated until } \\
\text { component failure }\end{array}$ \\
\cline { 2 - 3 } & Surface roughness: & $60 \mu \mathrm{mm}$ \\
\cline { 2 - 3 } & Surface treatment: & General surface treatment \\
\cline { 2 - 3 } & Material statistical influence: & $50 \%$ \\
\hline & Stress methodology & Critical plane method \\
\hline & Rainflow counting classes & $64 \times 64$ \\
\hline & Mean stress effect & Activated \\
\cline { 2 - 3 } & Damage for 4 laps (point A) & $\mathbf{0 , 0 0 6 2}$ \\
\hline & Life time in laps (point A) & $\mathbf{6 4 5}$ \\
\hline
\end{tabular}

Figure 11. Fatigue analysis settings and results

\section{CORRELATION WITH FAILURE}

After 702 laps at test track, a failure in the region A was found. As observed in figure 12, the failure was due crack propagation. Point $\mathrm{A}$ indicates the crack initiation region, the failure was due fatigue with multiple nucleation steps.

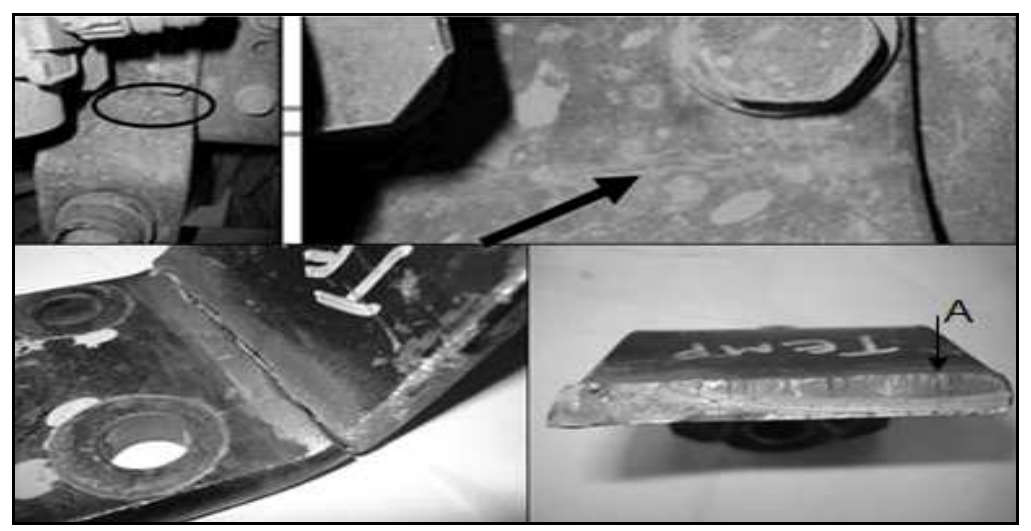

Figure 12. Failure by crack nucleation 
Comparing the damage prediction and the failure encountered, some observations can be made:

1. The FEA model and fatigue analysis software could represent the critical stress point, since the failure occurs at the region A.

2. The total time to failure is $8,5 \%$ higher than the life predicted with fatigue analysis.

The observation number 2 can be explained with the following equation:

$$
T_{T}=T_{f}+T_{p}
$$

Where:

$T_{T}=$ Total time to failure

$T_{f}=$ Time to crack nucleation

$T_{p}=$ Time to crack propagation until component inspection

Failure in life-time fatigue analysis is defined when a minimum crack is observed after the component been submitted to a number of cyclic loading [1]. Therefore, the life predicted in figure 11 can be compared only with the time $T_{f}$, and not with the total time $T_{T}$. The time $T_{p}$ can be calculated by the fracture mechanics and crack propagation approach, which is not the aim of the present study.

\section{COMPONENT SECOND VERSION DESIGN AND APPROVAL}

The component first version could not be homologated in the torture track due its failure. Thus, new component geometry was made, as showed in figure 13. It is important to notice that the main concept of the part was not changed, as well as, the load input region, and only additional material was introduced to reinforce the component.

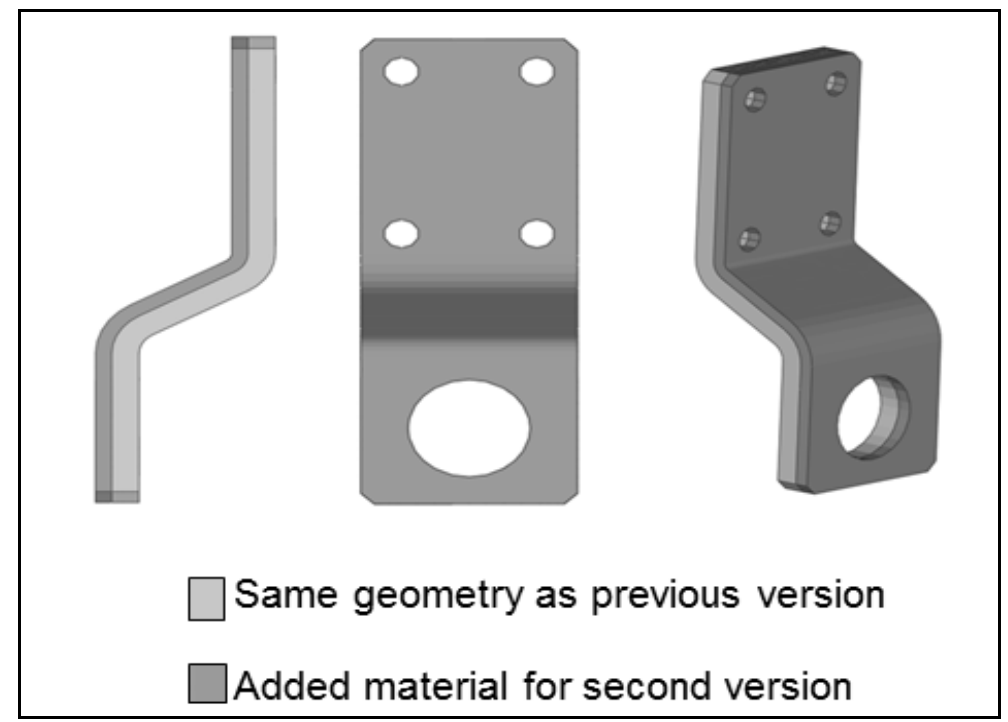

Figure 13. Component FEA model (second version) 
Following the same design process for this second version, the same FEA analysis is made, with the same torsion load case at the complete vehicle, but changing the component geometry. The new result is observed in figure 14.

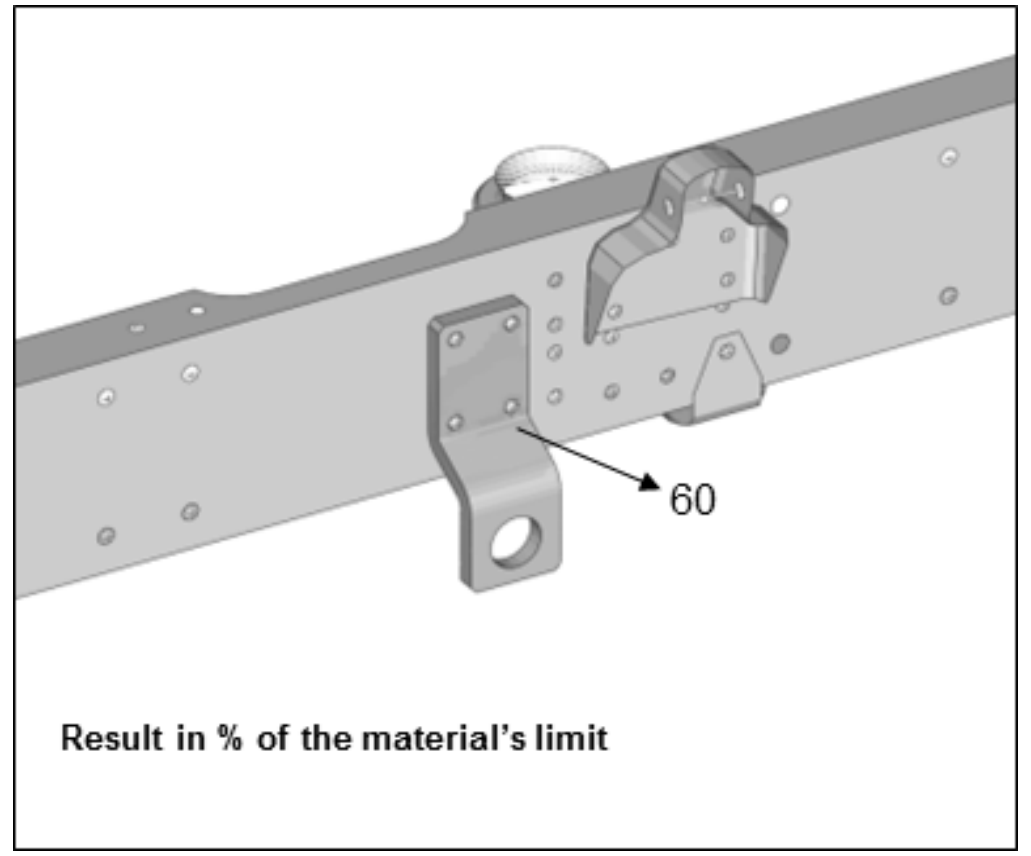

Figure 14. Stress results for torsion load case

At this step of the development process, another round of test in the accelerated durability proving ground should be necessary to approve the new component version. The present study aims to homologate the component second version without the extra round of test, approving it via FEA analysis, fatigue analysis and force measurement correlation. To make it possible, some assumptions have to be adopted:

1. The new geometry follows the same concept from the previous one, i.e. the critical points does not change.

2. The component load input direction and value cannot change between the first and second version.

The part studied in this paper follows the 2 assumptions previously described:

1. The first version concept is followed, shown in figure 13 and the critical points remain the same as shown in figure 14 .

2. Table 1 shows a comparison between the load input at torsion load case for each version. Since the mechanism that input the load into the component does not change, and the new geometry follows the same concept from the previous one, neither the value nor the direction of load has to have significant changes.

\begin{tabular}{|l|l|}
\hline First version load input (kgf) & 2294 \\
\hline Second version load input (kgf) & 2310 \\
\hline
\end{tabular}

Table 1. Comparison between the load inputs at FEA analysis (torsion load case) for each version 
Since the assumptions are valid, the same FEA and further fatigue analysis showed in figure 10 can be done, maintaining the reduced FEA model, the previous load measurement but now using the component second version. The parameters of the analysis, as well as, its results can be seen in figure 15 .

\begin{tabular}{|l|l|l|}
\hline \multirow{4}{*}{} & \multicolumn{2}{|c|}{ Fatigue analysis } \\
\cline { 2 - 3 } & Load input: & $\begin{array}{l}4 \text { laps extrapolated until } \\
\text { component failure }\end{array}$ \\
\cline { 2 - 3 } & Surface roughness: & $60 \mu \mathrm{mm}$ \\
\cline { 2 - 3 } & Surface treatment: & General surface treatment \\
\cline { 2 - 3 } & Material statistical influence: & $50 \%$ \\
\hline & Stress methodology & Critical plane method \\
\hline Rainflow counting classes & $64 \times 64$ \\
\hline & Mean stress effect & Activated \\
\cline { 2 - 3 } & Damage for 4 laps (point A) & $\mathbf{0 , 0 0 0 0 8 5}$ \\
\hline & Life time in laps (point A) & $\mathbf{4 7 0 0 0}$ \\
\hline
\end{tabular}

Figure 15. Fatigue analysis settings and results

By analyzing the second version part simulated life time it can be observed that it fulfills the approval criteria required to this torture track (2940 laps), with a safety factor of 15 . Therefore, the component second version does not need a second round of testing, and it is approved by fatigue simulation correlated with load measurement from former part version.

\section{CONCLUSION}

The presented study has covered the main steps of product development, giving a focus to time reduction during the component homologation aided by FEA analysis, force measurement correlation and fatigue analysis. Therefore, the following conclusions can be made:

1. The vehicle FEA has good correlation with reality, since it pointed the failure region as the component highest stress region.

2. The life time analysis has led to a good correlation between simulation and reality, it contributes to the parameter analysis assertiveness, such as the critical plane approach and fatigue analysis settings. But it is important to state that only one component failure was studied.

3. The good correlation rate between simulation and reality proves a satisfactory use of the load measurement extrapolation for fatigue analysis.

4. To improve the correlation, a fracture mechanics model could have been integrated to the fatigue analysis, this would cover the crack nucleation and propagation time.

5. To ensure the methodology effectiveness several assumptions have to be satisfied, such as: the new geometry has to follow the same concept from the previous one and the component load input direction and value cannot change between the versions. 


\section{REFERENCES}

[1] DOWLING, N. E. Mechanical Behavior of Materials, 3rd edition. Englewood Cliffs: Prentice Hall, 2007.

[2] QUEK, S. S.; LIU, G. R. Finite Element Method: A Practical Course. Oxford: Butterworth-Heinemann, 2003.

[3] Miner, M. A. Cumulative Damage in Fatigue. Journal of Applied Mechanics, v.12, p. 159-164, 1945.

[4] NEUBER, H. Theory of Stress Concentration for Shear-Strained Prismatical Bodies with Arbitrary Nonlinear Stress-Strain Law. Journal of Applied Mechanics, v.28, p. 544-550, 1961.

[5] FATEMI, A.; SHAMSAEI, N. Multiaxial Fatigue: An overview and some approximation models for life estimation. International Journal of Fatigue, v.33, p. 948-958, 2011.

[6] SPINELLI, D. M. "Lean development methodology for structural components development supported by design tools, manufacturing process and materials testing." São Carlos: Doctor Thesis - Escola de Engenharia de São Carlos, Universidade de São Paulo, 2012. [7] Gaier, C.; Dannbauer H. A Multiaxial Fatigue Analysis Method For Ductile, Semi-Ductile, And Brittle Materials. The Arabian Journal for Science and Engineering, v.33, p. 223 - 235, 2008.

[8] FKM-Guideline. Analytical Strength Assessment, Frankfurt am Main:

Forschungskuratorium Maschinenbau, 2003.

\section{DEFINITIONS}

$D=$ Total damage

$D_{i}=$ Damage for each different stress $\sigma_{i}$

$n_{i}=$ Number of cycles that a component is subjected in a given alternating stress $\sigma_{i}$.

$N_{i}=$ Number of cycles to failure at $\sigma_{i}$, according to component material's Wöhler curve.

$T_{T}=$ Total time to failure

$T_{f}=$ Time to crack nucleation

$T_{p}=$ Time to crack propagation until component inspection 